ISSN 1112-9867

http://www.jfas.info

\title{
INFLUENCE OF MAGNETIC FIELDS ON THE CRITICAL TEMPERATURE OF BULK YBCO SUPERCONDUCTOR WITH NANOMETER-SIZED AL $\mathrm{AL}_{2}$ INCLUSIONS
}

\author{
G. A. Shams ${ }^{1, *}$ and A. Mahmoodinezhad ${ }^{2}$ \\ ${ }^{1}$ Department of Physics, Shiraz Branch, Islamic Azad University, Shiraz, Iran \\ ${ }^{2}$ Department of Physics, Science and Research Branch, Islamic Azad University, Iran
}

Published online: 05 June 2016

\begin{abstract}
YBCO specimens along with different amounts of nano-sized $\mathrm{Al}_{2} \mathrm{O}_{3}$ were prepared using solid state reaction method. The structural characterization of all samples has been carried out by X-ray diffraction (XRD) and scanning electron microscopy (SEM) techniques. The XRD patterns with Rietveld refinement procedure was used to characterize the samples crystal microstructure. SEM analysis revels the phases with distribution of $\mathrm{Al}_{2} \mathrm{O}_{3}$ nanoparticles along the YBCO grain boundaries systematically. Superconducting parameters are affected by the nanoparticle addition, and results are discussed in bearing to the structural and microstructure differences between pure Y-123 and doped Y-123. The temperature dependence of electrical resistivity $\rho(\mathrm{T})$ has been measured and the effect of addition of $\mathrm{Al}_{2} \mathrm{O}_{3}$ nanoparticles with and without magnetic fields in the superconductivity and normal region has been reported.
\end{abstract}

Keywords: $\mathrm{YBa}_{2} \mathrm{Cu}_{3} \mathrm{O}_{7-\delta} ; \mathrm{Al}_{2} \mathrm{O}_{3}$ nanoparticles; critical temperature; magnetic fields.

Author Correspondence, e-mail: shams@iaushiraz.ac.ir, shams_physics@yahoo.com doi: http://dx.doi.org/10.4314/jfas.8vi2s.16 


\section{INTRODUCTION}

Superconductivity is a very outstanding and fascinating field of physics. Scientists and engineers throughout the world have been determined to develop an understanding of this interesting phenomenon. This phenomenon occurs in certain materials generally at low temperatures. The most important properties of these materials are: exact zero electrical resistance and the expulsion of the interior magnetic field or perfect diamagnetism (Meissner effect) [21]. These properties accompany with magnetic flux quantization and persistent current in superconductors are influenced by a very important factor named critical temperature $\left(T_{c}\right)$. At this temperature the conducting phase is in equilibrium with the superconducting phase. The zero electrical resistance property which deals with the flow of charged particles (electron pairs) without dissipation, combined with the discovery of high-temperature superconductors (HTSCs) in and after 1986 [5], began dreaming up practical applications in superconductivity at room temperature. The cuprate superconductors adopt a perovskite structure and are considered to be quasi two dimensional materials with their superconducting properties determined by electrons moving within weakly coupled copper-oxide $\left(\mathrm{CuO}_{2}\right)$ layers. Currently, the highest $T_{c}$ in the cuprate superconductor $\mathrm{Hg}-\mathrm{Ba}-\mathrm{Ca}-\mathrm{Cu}-\mathrm{O}$ is approximately equal to $135 \mathrm{~K}$ at $1 \mathrm{~atm}$ [28]. The discovery of a room temperature superconductor should trigger a great technological revolution. To enable the emerging new technologies, the superconducting materials with a superior performance can be developed by manipulating the appropriate "elementary building blocks" through nanostructuring. For superconductivity, such "elementary blocks" are Cooper pairs and fluxons [23] which is related to the magnetic flux. A more comprehensive study can be found in recent literatures $[1,10,36]$. A book with a discussion about room temperature superconductivity is available [24]. The knowledge of the microscopic mechanisms of oxide superconductors should be a theoretical guide in the researches to synthesize a room temperature superconductor. Superconducting-nanomaterial doped composites or some granular superconducting materials with weak-link characteristics can be regarded as those composed of superconducting grains embedded in a non-superconducting host. The latter can be a normal metal, an insulator, a ferromagnet, a semiconductor, or even a superconductor with lower transition temperature. As far as practical applications concern, literatures suggested that these materials may exhibit novel properties different from their pure superconducting phases $[9,14-15,25,30]$. One prominent feature 
of such materials is the existence of two superconductive transitions: a higher one and a lower one. At the higher one the grains become superconducting but the matrix remains normal and at the lower one the whole composite becomes superconducting but the critical current density $\left(J_{c}\right)$ is low. Another striking feature is that the magnetic flux pinning and critical current density of superconducting composites are enhanced at a low fraction of several non-superconductors [11, 18]. The most obvious application of these materials is to make a superconducting fault current limiter (SFCL) because composite superconductors have a broad range of current-carrying capacity [17]. Nowadays, studying on high temperature polycrystalline superconductor of $\mathrm{YBa}_{2} \mathrm{Cu}_{3} \mathrm{O}_{7-\delta}$ has stimulated great interest among researchers for most bulk applications at $77 \mathrm{~K}$ [33]. The investigation of various approaches to enhance the performance of high temperature superconductors for a variety of commercial applications requires enhanced electric current densities in high applied magnetic fields and temperatures. For polycrystalline $\mathrm{YBa}_{2} \mathrm{Cu}_{3} \mathrm{O}_{7-\delta}(\mathrm{YBCO}$ or $\mathrm{Y}-123)$, magnetic field penetrates into the material as an array of quantized vortices or flux lines, and the magnitude of $J_{c}$ extremely depends on how effectively these flux lines are pinned by lattice defects in the superconducting matrix [2]. Nanoparticles addition such as $\mathrm{BaZrO}_{3}$ [31], $\mathrm{ZrO}_{2}$ [37], $\mathrm{ZnO}$ [39], $\mathrm{SnO}_{2}$ [38] and $\mathrm{Al}_{2} \mathrm{O}_{3}$ can react with superconductor materials like YBCO during the manufacturing process that some of them have been reported to have significant effect on superconductors critical parameters specifically increasing of $J_{c}$. In this paper, we have added different amounts of nano-sized alumina $\left(\mathrm{Al}_{2} \mathrm{O}_{3}\right)$ to the bulk $\mathrm{YBCO}$ superconductor or Y-123 for investigation of the critical temperature parameter $T_{c}$ in presence of magnetic fields up to $400 \mathrm{mT}$. The measurements were done using standard four-probe technique. Phase identification of specimens was obtained by X-ray with the Rietveld refinement diffraction method. SEM analysis demonstrates the samples crystal structure and distribution of $\mathrm{Al}_{2} \mathrm{O}_{3}$ nanoparticles along the YBCO grain boundaries.

\section{EXPERIMENTAL}

Electrical, magnetic and thermal properties of high temperature superconductors are related to the purity and processing condition of the samples. Many papers have been published on sample processing for the fabrication of high quality superconductors [4, 8, 12, 19]. Most attention in this arena has been on the (YBCO) superconductor in order to optimize the purity 
of the superconducting phase. Among the three most common methods for the preparation of YBCO samples involved the fabrication of a pressed polycrystalline disc, flux growth of single crystals [13] and melt-textured growth (MTG) crystal [16, 20] the pressed disc polycrystalline $\mathrm{YBa}_{2} \mathrm{Cu}_{3} \mathrm{O}_{7-\delta}$ (Y-123) samples were chosen and prepared by using the standard solid state reaction method [7]. This method allows off-the-shelf high purity chemicals, the $\mathrm{YBa}_{2} \mathrm{Cu}_{3} \mathrm{O}_{7-\delta}$ and $\mathrm{Al}_{2} \mathrm{O}_{3}$ nanoparticles doping samples to be fabricated and processed into ceramic, polycrystalline materials. The phase diagram of YBCO using the base compounds $\mathrm{Y}_{2} \mathrm{O}_{3}, \mathrm{BaO}$ and $\mathrm{CuO}$ is shown by the work of Taylor et al. [34], where the superconducting Y-123 and insulating Y-211phases are distinguished. Alumina nanopowder (20 $\mathrm{nm}$ in diameter which is determined by transmission electron microscopy (TEM)) with different amounts of $0.0,0.06,0.1$ and $0.3 \mathrm{wt} \%$ were added and synthesized to $\mathrm{YBa}_{2} \mathrm{Cu}_{3} \mathrm{O}_{7-\delta}$ using conventional solid state reaction method. The powders were pressed into pellets at 500 $\mathrm{MPa}$ in the form of circular discs with dimensions of $30 \mathrm{~mm}$ in diameter and $4 \mathrm{~mm}$ in thickness. The calcination process involved heating the furnace to $750^{\circ} \mathrm{C}$ at the rate of $200^{\circ}$ $\mathrm{C} / \mathrm{hr}$ and soaking for twelve hours, followed by heating to $930^{\circ} \mathrm{C}$ at the rate of $150^{\circ} \mathrm{C} / \mathrm{hr}$ and then calcining at $930^{\circ} \mathrm{C}$ for twenty-four hours before cooling to room temperature at the rate of $60^{\circ} \mathrm{C} / \mathrm{hr}$. The calcined pellets were not of good quality and can be easily broken and divided into layers, therefore a sintering process was necessary. For the sintering process, the calcined samples were first ring milled for twenty minutes, the particles being $<10 \mu \mathrm{m}$ in size, and the powder was then pressed into pellets using again the $30 \mathrm{~mm}$ diameter hardened steel die and uniaxially pressure of 400 bar to obtain approximately a $3 \mathrm{~mm}$ thick disk. The pellets were put into the furnace and the temperature increased to $930^{\circ} \mathrm{C}$ at the rate of $90^{\circ} \mathrm{C}$ per hour. The pellets were sintered at this temperature for 24 hours. The temperature was then decreased to $720^{\circ} \mathrm{C}$ at a rate of $20^{\circ} \mathrm{C}$ per hour, followed by cooling to room temperature in the presence of 1 atmosphere oxygen at the rate of $10^{\circ} \mathrm{C}$ per hour. We considered pure powder $(0$ $\mathrm{wt} \%$ ) as a reference, it was hand ground just as the $\mathrm{Al}_{2} \mathrm{O}_{3}$ doped samples to ensure identical conditions due to physical properties of the samples. After preparation the structure and phase identification of $\mathrm{Y}-123$ samples doped and undoped with nano-sized $\mathrm{Al}_{2} \mathrm{O}_{3}$ were characterized by powder X-ray diffraction (XRD) using a Bruker D 8 Advance diffractometer with $\mathrm{CuK} \alpha$ 
radiation. The morphology and microstructures of specimens was specified by scanning electron microscope (SEM) using KYKY-EM3200. The transport properties of the samples were studied by measuring the electrical resistivity temperature $\rho(\mathrm{T})$ using the four-probe technique. The pellets were carefully cut into bar-shaped samples with almost similar dimensions. Electrical contacts were made using silver paint and the contact resistance value was approximately $0.5 \mathrm{ohm}$. A low excitation current $100 \mathrm{~mA}$ is used in order not to affect the behavior of the resistivity transition of the samples. In order to determine acceptable accuracy, the acquisition of the data close to the transition was obtained with the temperature varying in rates of $4 \mathrm{~K} / \mathrm{h}$ and two $\rho(\mathrm{T})$ runs, one for decreasing temperatures, the other for increasing temperatures were accomplished.

\section{RESULTS AND DISCUSSION}

The XRD patterns of pure Y-123 along with nano-sized $\mathrm{Al}_{2} \mathrm{O}_{3}$ doping up to $0.3 \mathrm{wt} \%$ have been shown in figure 1. It is clear from the Rietveld refinement method that no observed any peak related to the alumina except YBCO phase which is detected in the X-ray patterns. The lattice constant parameters for prepared specimens are nearly same with average values of $a=3.821 \AA, b=3.880 \AA$ and $c=$ $11.663 \AA$ which are comparable with the literature for undoped Y-123 [6], but it is expected that the $a$ and $c$ parameters increase slightly in higher amounts of $\mathrm{Al}_{2} \mathrm{O}_{3}$ content whereas $b$ parameter remains constant. These alterations illustrate that the $\mathrm{Al}^{3+}$ ions occupy both of $\mathrm{Y}$ and $\mathrm{Cu}$ (1) sites. According to the reflections related to the XRD patterns $(h k l)$ can be deduce that (013) and (103) peaks merged together and with increment of $\mathrm{Al}_{2} \mathrm{O}_{3}$ nanoparticles the (103) reflection tends to lower angle which is caused orthorhombicity of the system decreased [22]. 


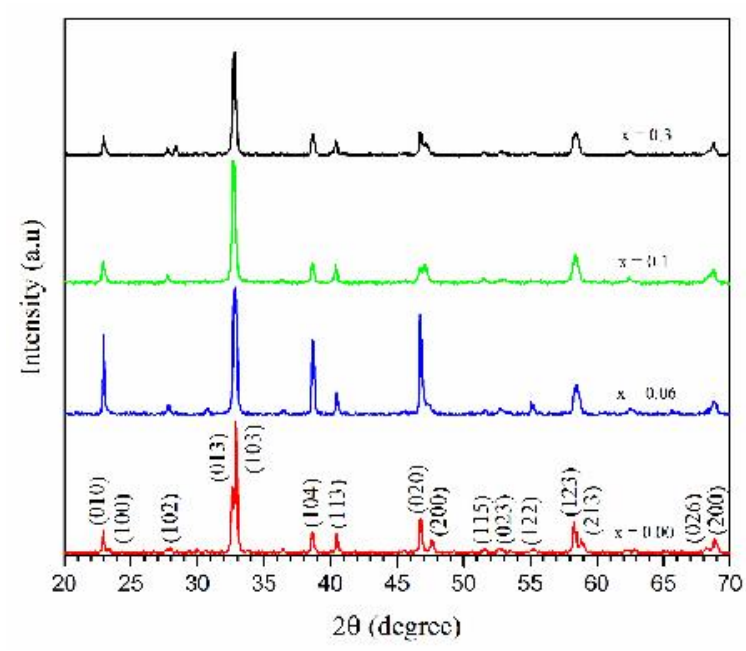

Fig.1. XRD patterns of $\mathrm{Y}-123$ samples with $\mathrm{x}=0.00$ to $\mathrm{x}=0.3 \mathrm{wt} \% \mathrm{Al}_{2} \mathrm{O}_{3}$ nanoparticles doping

The relationship between electrical resistivity-temperature for undoped and doped YBCO samples with different amounts of nano-sized $\mathrm{Al}_{2} \mathrm{O}_{3}$ has been shown in figure 2 . In this figure the general features of the temperature dependence of the resistivity is the same as the data reported previously $[29,35]$ which has shown that the high- $T_{c}$ superconducting samples are generally characterized by a linear temperature dependence over a large temperature range, unless the stoichiometric composition of the sample differs significantly [26-27]. Presented figure demonstrates the superconducting transition temperature $T_{c o}$, particularly for pure polycrystalline Y-123 sample is sharp enough and clear. $T_{c o}$ value is taken from where an extrapolation of the superconducting transition intercepts the zero axes, the foot of curve is not considered. The resistivity value is $91.00 \mathrm{~K}, 84.50 \mathrm{~K}, 79.50 \mathrm{~K}$ and $77.10 \mathrm{~K}$ corresponding to the specimens with $\mathrm{x}=0, \mathrm{x}=0.06, \mathrm{x}=0.1$ and $\mathrm{x}=0.3 \mathrm{wt} \%$ respectively. These changes indicate that with increment of nano-alumina content the $T_{c o}$ decline considerably. With regard to the critical temperature values and crystal structure data, the occupation of $\mathrm{Y}$ and $\mathrm{Cu}$ (1) sites by $\mathrm{Al}^{3+}$ ions impress the $T_{c}$ of samples and results in degradation of superconductivity that is in agreement with Antal et al. [3]. The value of superconducting transition temperatures $T_{c o}$, for the pure $(\mathrm{x}=0.00)$ polycrystalline $\mathrm{Y}-123$ material, determined from the resistivity curve, again ignoring the foot structure, is significantly higher than those obtained from another method such as thermoelectric power curves. This result is not unexpected as the two methods used to determine $T_{c o}$ depend on different physical processes. 
Once there is a sufficient density of Cooper pairs to carry the measurement current in the resistivity determination, the remaining normal electron resistivity is shorted-out and $T_{c o}$ is obtained. In the case of thermoelectric power measurements, charge separation continues past the initial condensation process as the normal electron density must be reduced significantly before charge separation goes to zero. The temperature gradient is still maintained primarily by the phonon conductivity as the Cooper pairs do not transfer heat. The resistivity curves in the metallic regime can be expressed by the linear equation $\rho_{n}(T)=\rho_{0}+\alpha T$, where $\alpha$ is the resistivity temperature coefficient, considered as a parameter that depends on the intrinsic electronic interaction and $\rho_{0}$ is the residual resistivity considered as an indicator of the sample homogeneity and defect density. All of the resistivity is $\geq 2$ times larger than for resistivity of high purity bulk Y-123 with optimal doping at $91 \mathrm{~K}$ [32]. The normal state resistivity increases with increasing $\mathrm{Al}_{2} \mathrm{O}_{3}$ concentration in all samples. To account for this increase in resistivity it is assumed that the dominant process involves increased electron scattering from both the small and large angle grain boundaries associated with the different crystallographic orientations. The absolute resistivity in the normal state depends on porosity, grain boundary scattering, and its linearity over a longer temperature interval confirms the idea that the preparation and synthesis of the samples are done correctly, i.e., with optimal oxygen content. As is seen in figure 2 the onset of $T_{c}$ with increasing of $\mathrm{Al}_{2} \mathrm{O}_{3}$ nanoparticles decline slightly. For doped samples with different amounts of nano-alumina up to $0.3 \mathrm{wt} \%$ the $T_{c}$ is somewhat lower than undoped sample means pure Y-123 that begins to decline sharply near $92 \mathrm{~K}$ and reaches zero near $91 \mathrm{~K}$.

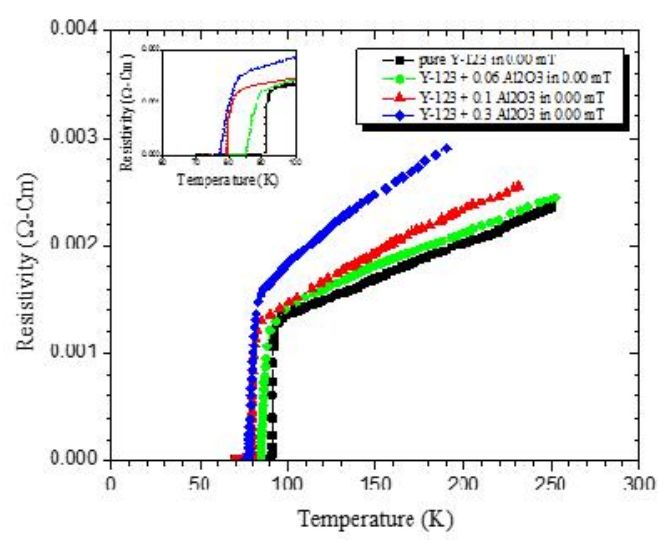

Fig.2. Critical temperature $T_{c}$ of the Y-123 samples with addition of $\mathrm{x}=0.0$ to $\mathrm{x}=0.3$ nano-alumina without applied magnetic fields 
The $T_{c}$ of specimens with and without applied magnetic fields related to $\mathrm{x}=0$ to $\mathrm{x}=0.3 \mathrm{wt} \%$ doping is presented in table 1 . With respect to the data can be expressed that without applied magnetic fields below $79.00 \mathrm{~K}$ the whole of samples would be superconductors and higher resistivity occurs in normal state for upper dopant. For applied magnetic fields cases, the data changes to $71.50 \mathrm{~K}$ and $67.50 \mathrm{~K}$ for $200 \mathrm{mT}$ and $400 \mathrm{mT}$ respectively. These results clearly indicate that magnetic fields regress the critical superconducting temperature.

Table 1. Transition temperature associated with resistivity measurement in the vicinity of applied magnetic fields $(0.00 \mathrm{mT}, 200 \mathrm{mT}$ and $400 \mathrm{mT})$ for YBCO samples with different amounts of $\mathrm{Al}_{2} \mathrm{O}_{3}$ nanoparticles addition

\begin{tabular}{|c|c|c|c|c|c|}
\hline Samples & $\mathrm{x}$ & $\begin{array}{c}\text { Onset } \\
\text { transition } \\
\text { temperature } \\
T_{c o}(\mathrm{~K}) \text { in zero } \\
\text { field }\end{array}$ & $\begin{array}{c}\text { Critical } \\
\text { superconducting } \\
\text { temperature } T_{c} \\
(\mathrm{~K}) \quad \text { in zero } \\
\text { field }\end{array}$ & $\begin{array}{c}\text { Critical } \\
\text { superconducti } \\
\text { ng } \\
\text { temperature } \\
T_{c}(\mathrm{~K}) \text { in } 200 \\
\mathrm{mT}\end{array}$ & $\begin{array}{c}\text { Critical } \\
\text { superconducti } \\
\text { ng temperature } \\
T_{c}(\mathrm{~K}) \text { in } 400 \\
\mathrm{mT}\end{array}$ \\
\hline \multirow{4}{*}{$\begin{array}{c}\mathrm{Y}-123+\mathrm{x} \\
\mathrm{wt} \% \\
\mathrm{Al}_{2} \mathrm{O}_{3}\end{array}$} & 0.00 & 91.00 & 92.20 & 87.50 & 84.50 \\
\hline & 0.06 & 84.50 & 86.70 & 81.60 & 78.80 \\
\hline & 0.10 & 79.50 & 82.60 & 75.50 & 71 \\
\hline & 0.30 & 77.10 & 79.00 & 71.50 & 67.50 \\
\hline
\end{tabular}

The temperature dependence of the electrical resistivity $\rho(T)$ in the vicinity of applied magnetic fields $200 \mathrm{mT}$ and $400 \mathrm{mT}$ for $\mathrm{YBa}_{2} \mathrm{Cu}_{3} \mathrm{O}_{7-\delta}$ polycrystalline sintered with different amounts $(0.0,0.06,0.1$ and $0.3 \mathrm{wt} \%)$ of $\mathrm{Al}_{2} \mathrm{O}_{3}$ nanoparticles are shown in Fig. 3a, and b, respectively. All samples exhibit metallic-like behavior in the normal state at the presence of applied magnetic fields $200 \mathrm{mT}$ and $400 \mathrm{mT}$, with an increase resistivity at room temperature. According to figure 3 the different resistivity at room temperature, followed by a superconducting transition at $T_{c o}$ which is highly dependent on not only amounts of dopant but also magnetic fields. Value of $T_{c}$ is approximately 87.5 and $84.5 \mathrm{~K}$ for free added Y-123 with applied magnetic fields $200 \mathrm{mT}$ and 400mT, respectively, and is suppressed with increasing the amount of $\mathrm{Al}_{2} \mathrm{O}_{3}$ in all samples. The suppression of $T_{c}$ is consistent with the known behavior of the bulk superconductors when adding small amounts of impurities. The decrease in $T_{c}$ can thus be explained as arising due to $\mathrm{Al}_{2} \mathrm{O}_{3}$ induced modification of the grain 
boundary characteristics and progressively decoupling of the superconducting grains. A grain boundary is a region of structural distortions, where an extra energy appears; in order to decrease their energy, they tend to attract impurity atoms. $T_{c}$ decreases with increasing $\mathrm{Al}_{2} \mathrm{O}_{3}$ content and applied magnetic fields suggesting intragranular changes due to the incorporation of alumina.
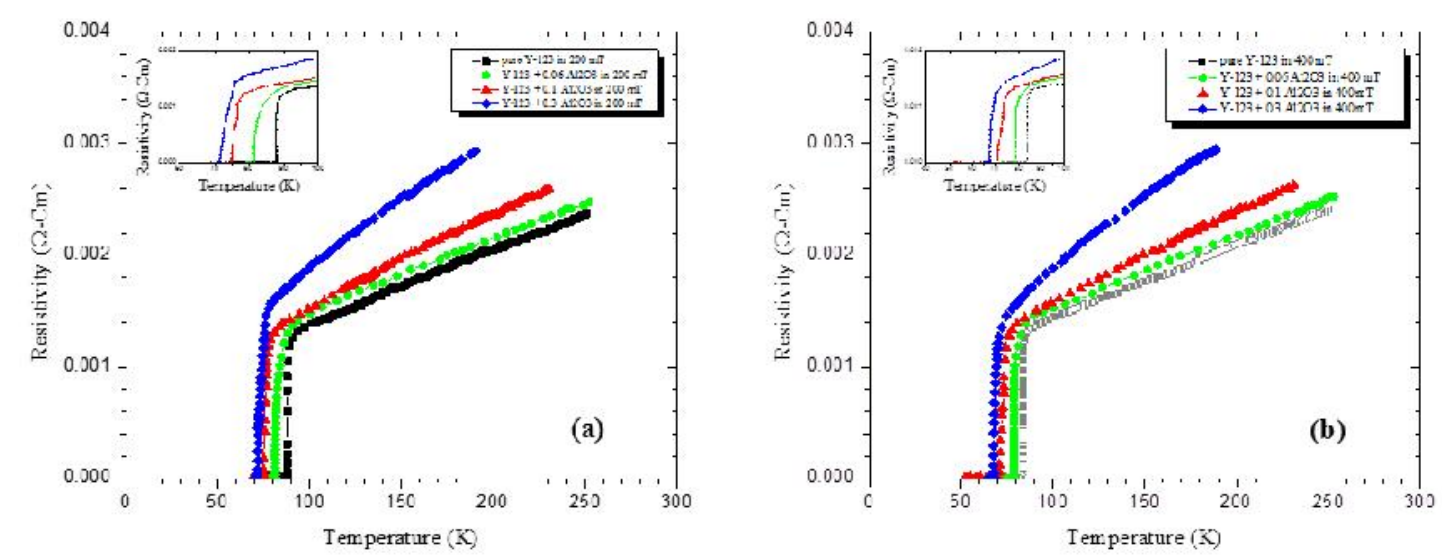

Fig.3. Different amounts of nano alumina doped on YBCO specimens in 200 \& $400 \mathrm{mT}$ applied magnetic fields

The resistivity as a function of temperature, with and without applied magnetic field, for polycrystalline Y123 samples are presented in Figures 4a to 4d. The application of magnetic fields to the polycrystalline samples, either field cooled (FC) or zero field cooled (ZFC), always decreases the value of the critical temperature $T_{c}$ for the superconductors below $T_{c}$, see figures 4.a to 4.d. This is expected as the thermal conductivity of superconductors is sensitive to the presence of magnetic vortices due to both carrier and phonon vortex. For temperatures above $T_{c}$, the fields $0.00 \mathrm{mT}, 200 \mathrm{mT}$ and $400 \mathrm{mT}$ were found not to alter the normal state curves regarding the shape and values for samples with $0.0,0.06$ and 0.1 wt $\%$ of $\mathrm{Al}_{2} \mathrm{O}_{3}$ nanoparticles inclusions, significantly (figures 4.a, 4.b, and 4.c). This scenario is different for the sample with $0.3 \mathrm{wt} \%$ not only due to the shape but also the value (figures 4.d). Figure 4.d clearly shows field changes for the doped content $0.3 \mathrm{wt} \%$. The maximum change occurs for the magnetic field $400 \mathrm{mT}$, which is related to the doping value of $0.3 \mathrm{wt} \% \mathrm{Al}_{2} \mathrm{O}_{3}$ nanoparticles. Additional magnetic field results can be discussed if the pinning properties effect is considered. 

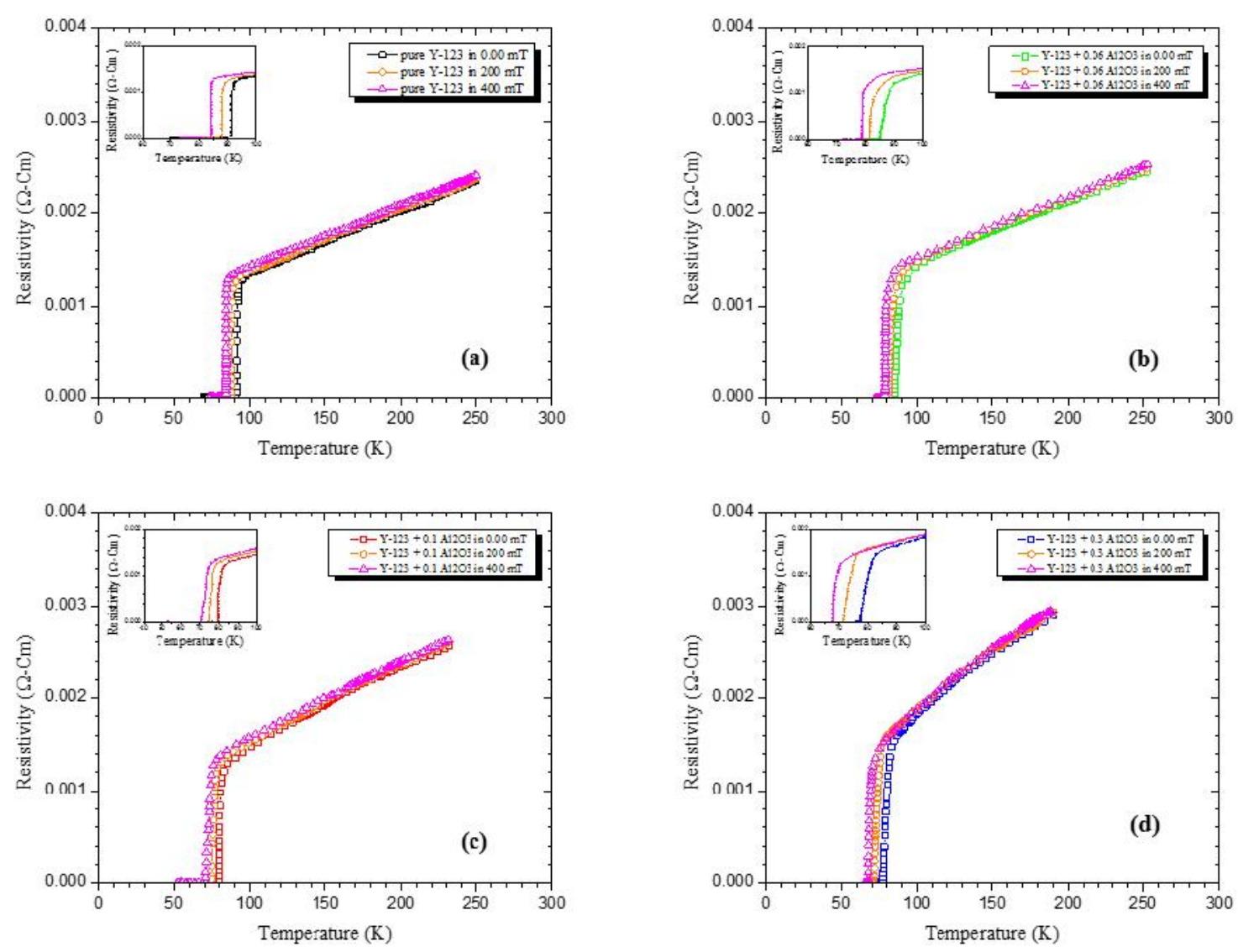

Fig.4. $T_{c}$ of the YBCO samples with various values of nano-sized $\mathrm{Al}_{2} \mathrm{O}_{3}$ in different applied magnetic fields (a) $0.00 \mathrm{mT}$, (b) $200 \mathrm{mT}$ and (c) $400 \mathrm{mT}$

Evolutions and regressions of the residual resistivity $\rho_{o}$ as a function of various values of $\mathrm{Al}_{2} \mathrm{O}_{3}$ nanoparticles in the vicinity of applied magnetic fields $(200 \mathrm{mT}$ and $400 \mathrm{mT})$ for YBCO samples is shown in figure 5. The parameter $\rho_{\mathrm{o}}$ increases almost rapidly at the same slope for both cases of $200 \mathrm{mT}$ and $400 \mathrm{mT}$ applied magnetic fields for added Y123 sample $0.06 \mathrm{wt} \%$. This process followed by decrease rapidly in residual resistivity for added $0.01 \mathrm{wt} \%$ sample due to applied magnetic fields. The increase in the $\rho_{\mathrm{o}}$ can be understood by the decrease of the relaxation of time due to a greater number of defects, disorder and inhomogeneities as the $\mathrm{Al}_{2} \mathrm{O}_{3}$ concentration increases. By increasing of $\mathrm{Al}_{2} \mathrm{O}_{3}$ doping to $0.3 \mathrm{wt} \%$ the regressions of the residual resistivity for $400 \mathrm{mT}$ applied magnetic field will continue, while for $200 \mathrm{mT}$ the evolutions of the residual resistivity occur. This process might be explained as follows: $\mathrm{CuO} 2$ planes adjacent to the charge reservoir layers have a pyramidal oxygen coordination and are over-doped, while the $\mathrm{CuO} 2$ plane with a square oxygen coordination is under-doped. This disparity in the carrier concentration might be one of the 
causes of the higher normal-state resistivity observed in added Y123 sample 0.3wt\% when applied magnetic field was $400 \mathrm{mT}$. The critical temperature $T_{c}$ behavior declined slightly in an applied magnetic fields for whole of $\mathrm{YBCO}$ doped samples with $\mathrm{Al}_{2} \mathrm{O}_{3}$ nanoparticles. However, the magnetic fields applied to the superconducting Y123 pure and added $\mathrm{Al}_{2} \mathrm{O}_{3}$ nanoparticles samples produce significant effect on the electrical resistivity curves and critical temperatures.

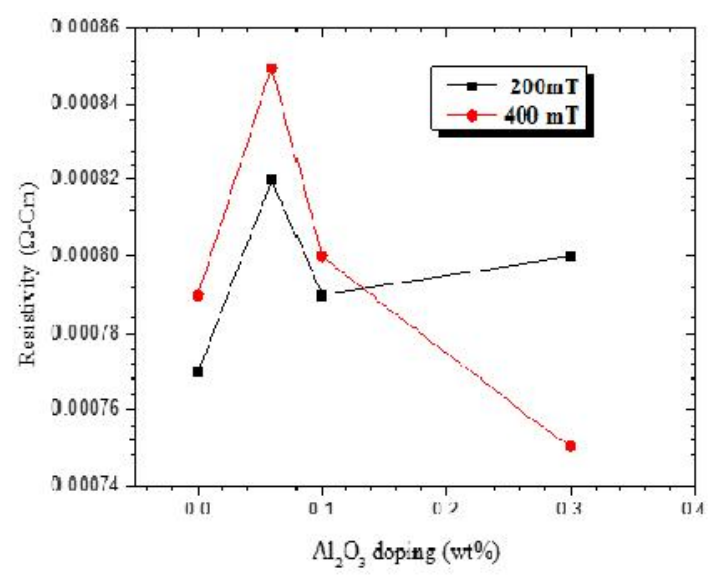

Fig.5. Evolutions and regressions of the residual resistivity $\rho_{\mathrm{o}}$ of Y-123 samples as a function of various amounts of nano-sized $\mathrm{Al}_{2} \mathrm{O}_{3}$ particles in the vicinity of $200 \mathrm{mT}$ and $400 \mathrm{mT}$ magnetic fields

The microstructure of YBCO samples with added and not added nano-alumina are indicated in figure 6. With respect to the SEM images is observed that nano-sized $\mathrm{Al}_{2} \mathrm{O}_{3}$ have been distributed among $\mathrm{YBa}_{2} \mathrm{Cu}_{3} \mathrm{O}_{7-\delta}$ grain boundaries. Higher doping values of $\mathrm{Al}_{2} \mathrm{O}_{3}$ nanoparticles is caused to form the Al-rich region which dispersed into the Y-123 system and attach YBCO grains together such as connector [33]. The synthesized $\mathrm{YBa}_{2} \mathrm{Cu}_{3} \mathrm{O}_{7-\delta}$ samples prepared via the solid state reaction method with $\mathrm{Al}_{2} \mathrm{O}_{3}$ nanoparticles is composed of nanomaterial with homogenous distribution. The random orientation of grains and almost poor grain contact in some sections is evident. It is expected that with the decrease in grain size, the number of grain boundaries increases; a higher number of grain boundaries imply a greater number of weak links and consequently lower dimensional effects in the transport properties. 


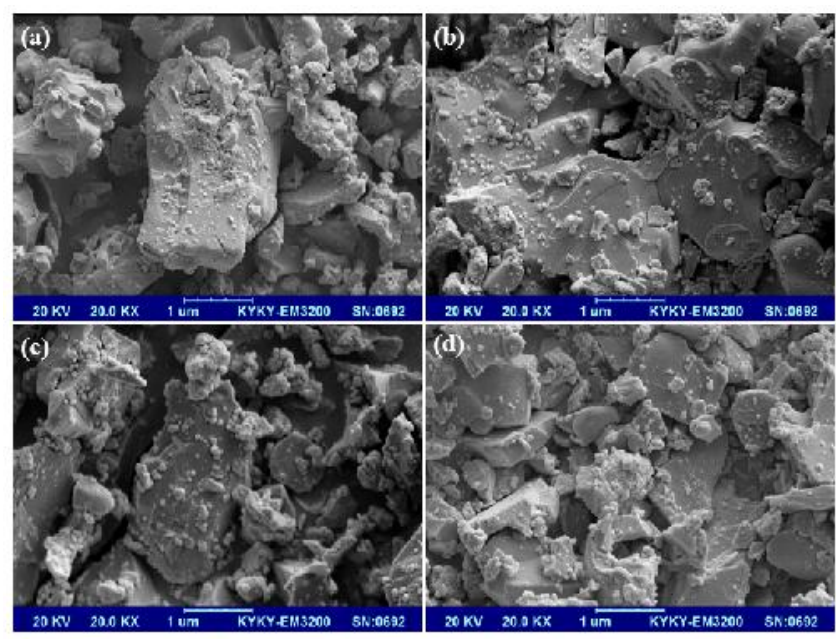

Fig.6. Microstructures of polycrystalline YBCO with (a) 0.0 , (b) 0.06 , (c) 0.1 and (d) 0.3 wt\% nanometer alumina particles doping

\section{CONCLUSION}

The influence of magnetic fields on critical temperature $T_{c}$ of bulk $\mathrm{YBa}_{2} \mathrm{Cu}_{3} \mathrm{O}_{7-\delta}$ superconductor with different amounts of nano-sized $\mathrm{Al}_{2} \mathrm{O}_{3}$ addition has been investigated. Specimens are blended using standard solid state reaction technique. Aluminum Oxide Nanopowder (nano-alumina) with size of $20 \mathrm{~nm}$ in diameter which is determined by transmission electron microscopy (TEM) were added to the Y-123 powder by mixing and hand grinding in an agate mortar along with pestle. The temperature dependence of electrical resistivity $\rho(\mathrm{T})$ has been measured and the effect of addition of $\mathrm{Al}_{2} \mathrm{O}_{3}$ nanoparticles in the superconductivity and normal region has been reported and the results have been discussed in terms of suppression with and without magnetic field. The critical temperature $T_{c}$ behavior declined slightly in an applied magnetic fields for whole of YBCO doped samples with $\mathrm{Al}_{2} \mathrm{O}_{3}$ nanoparticles. XRD patterns corresponding to the samples have indicated that $\mathrm{Al}^{3+}$ ions occupy both of $\mathrm{Y}$ and $\mathrm{Cu}$ (1) sites. With regard to SEM analysis can be deduced that nano-alumina well distributed in polycrystalline YBCO grain boundaries and same as bridge attach each grain together. However, the magnetic fields applied to the superconducting Y123 pure and added $\mathrm{Al}_{2} \mathrm{O}_{3}$ nanoparticles samples produce significant effect on the electrical resistivity curves and critical temperatures. 


\section{ACKNOWLEDGEMENTS}

The authors are willing to express their appreciation to Mr. Fatemi and Dr. Ghaffary from Islamic Azad University-Shiraz branch for their help and valuable comments. We are also grateful to research center, Shiraz Branch, Islamic Azad University, Shiraz, Iran for their financial support.

\section{REFERENCES}

[1] Aladyshkin, A. Y., Silhanek, A. V., Gillijns, W. and Moshchalkov, V. V. Nucleation of superconductivity and vortex matter in superconductor-ferromagnet hybrids. Superconductor Science \& Technology, 2009, Vol. 22. No. 5. 48.

[2] Albiss, B. A., Al-Rawashdeh, N., Abu Jabal, A., Gharaibeh, M., Obaidat, I. M., Hasan, M. K. and Azez, K. A. Polycrystalline YBa2Cu3O7-delta with Nano-sized Al2O3 Inclusions. Journal of Superconductivity and Novel Magnetism, 2010, Vol. 23. No. 7. 1333-1340.

[3] Antal, V., Kanuchova, M., Sefcikova, M., Kovac, J., Diko, P., Eisterer, M., Horhager, N., Zehetmayer, M., Weber, H. W. and Chaud, X. Flux pinning in Al doped TSMG YBCO bulk superconductors. Superconductor Science \& Technology, 2009, Vol. 22. No. 10. 7.

[4] Balestrino, G., Barbanera, S. and Paroli, P. Growth of single-crystals of the high-temperature superconductor YBA2CU3O7-X. Journal of Crystal Growth, 1987, Vol. 85. No. 4. 585-587.

[5] Bednorz, J. G. and Muller, K. A. Possible high-tc superconductivity in the ba-la-cu-o system. Zeitschrift Fur Physik B-Condensed Matter, 1986,Vol. 64. No. 2. 189-193.

[6] Chen, C., Wondre, F.R., Hodby, J.W., Ryan, J.F., Narlikar, A.V. and Samanta, S.B. Relationship between Growth, Structure and Superconductivity of Single Crystal $\mathrm{YBa}_{2} \mathrm{Cu}_{3} \mathrm{O}_{7-\delta}$. Journal of Low Temperature Physics, 1999, Vol. 117. No. 3. 711-715.

[7] Costa, C. A., Ferretti, M. and Olcese, C. L. Synthesis of $\mathrm{YBa}_{2} \mathrm{Cu}_{3} \mathrm{O}_{7-x}$ polycrystalline superconductors from Ba peroxide: First physico-chemical characterization. Journal of Crystal Growth, 1987, Vol. 85. No. 4. 623-627. 
[8] Franck, J. P., Jung, J. and Mohamed, M. A. K. Superconductivity in the system $\left(\mathrm{Al}_{\mathrm{x}} \mathrm{Y}_{1-\mathrm{x}}\right) \mathrm{Ba}_{2} \mathrm{Cu}_{3} \mathrm{O}_{6.5+\delta}$. Physical Review B, 1987, Vol. 36. No. 4. 2308-2310.

[9] Gillijns, W., Aladyshkin, A. Y., Silhanek, A. V. and Moshchalkov, V. V. Magnetic confinement of the superconducting condensate in superconductor-ferromagnet hybrid composites. Physical Review B, 2007, Vol. 76. No. 6. 4.

[10]Hanggi, P. and Marchesoni, F. Artificial Brownian motors: Controlling transport on the nanoscale. Reviews of Modern Physics, 2009, Vol. 81. No. 1. 387-442.

[11]Huang, S. L., DewHughes, D., Zheng, D. N. and Jenkins, R. Effects of MgO addition on phase evolution and flux pinning of $\mathrm{Bi}-2212 / \mathrm{Ag}$ tapes fabricated by electrophoretic deposition and partial-melting processing. Superconductor Science \& Technology, 1996, Vol. 9. No. 5. 368-373.

[12]Hunter, BA, Town, SL, Matthews, DN, Russell, GJ and Taylor, KNR. Growth and Properties of Large Single Crystals of $\mathrm{YBa} 2 \mathrm{Cu} 3 \mathrm{O} 7-\mathrm{d}$ from $\mathrm{BaCuO} 2 / \mathrm{CuO}$ Flux. Australian Journal of Physics, 1991, Vol. 44. No. 4. 421-428.

[13]Ito, T., Takenaka, K. and Uchida, S. Systematic deviation from t-linear behavior in the inplane resistivity of yba2cu3o7-y - evidence for dominant spin scattering. Physical Review Letters, 1993, Vol. 70. No. 25. 3995-3998.

[14]John, S. and Lubensky, T. C. Spin-glass state of a randomly diluted granular superconductor. Physical Review Letters, 1985, Vol. 55. No. 9. 1014-1017.

[15]Repeated Author. Phase-transitions in a disordered antigranulocytes superconductor near percolation. Physical Review B, 1986, Vol. 34. No. 7. 4815-4825.

[16]Mahmoud, A. S. and Russell, G. J. Large crystals of the composite Y/Nd(123) containing various dopants grown by melt-processing in air. Superconductor Science \& Technology, 1998, Vol. 11. No. 10. 1036-1040.

[17]Mamalis, A. G., Ovchinnikov, S. G., Petrov, M. I., Balaev, D. A., Shaihutdinov, K. A., Gohfeld, D. M., Kharlamova, S. A. and Vottea, I. N. Composite materials on high-T-c superconductors and BaPbO3, Ag basis. Physica C, 2001, Vol. 364. No. 174-177.

[18]Matsumoto, K., Takewaki, H., Tanaka, Y., Miura, O., Yamafuji, K., Funaki, K., Iwakuma, M. and Matsushita, T. Enhanced jc properties in superconducting nbti 
composites by introducing nb artificial pins with a layered structure. Applied Physics Letters, 1994, Vol. 64. No. 1. 115-117.

[19]Matthews, D. N., Bailey, A., Vaile, R. A., Russell, G. J. and Taylor, K. N. R. Increased Transition-Temperatures IN YBA2CU3OY Superconducting Ceramics By Exposure To Nitrogen. Nature, 1987, Vol. 328. No. 6133. 786-787.

[20]Matthews, D. N., Cochrane, J. W. and Russell, G. J. Melt-Textured Growth AND Characterization of a (ND/Y)BA2CU3O7-Delta Composite Superconductor With Very High Critical-Current Density. Physica C, 1995, Vol. 249. No. 3-4. 255-261.

[21]Meissner, W and Ochsenfeld, R. Ein neuer Effekt bei Eintritt der Supraleitfahigkeit. Naturwiss, 1933, Vol. 21. No. 787-788.

[22]Mellekh, A., Zouaoui, A., Ben Azzouz, E., Annabi, A. and Ben Salem, A. Nano- $\mathrm{Al}_{2} \mathrm{O}_{3}$ particle addition effects on $\mathrm{YBa}_{2} \mathrm{Cu}_{3} \mathrm{O}_{\mathrm{y}}$ superconducting properties. Solid State Communications, 2006, Vol. 140. No. 6. 318-323.

[23] Moshchalkov, V, Wordenweber, R. and Lang, W. Nanoscience and Engineering in Superconductivity. Springer-Verlag Berlin Heidelberg, 2010.

[24]Petrov, M. I., Balaev, D. A., Gohfeld, D. M., Ospishchev, S. V., Shaihudtinov, K. A. and Aleksandrov, K. S. Applicability of the theory based on Andreev reflection to the description of experimental current-voltage characteristics of polycrystalline HTSC plus normal metal composites. Physica C, 1999, Vol. 314. No. 1-2. 51-54.

[25]Phillips, J. C. Reconciliation of Normal-State and Superconductive Specific-Heat, Optical, Tunneling, and Transport Data on Y-BA-CU-O. Physical Review B, 1989, Vol. 40. No. 10. 7348-7349.

[26]Sahoo, M and Behera, D. Effect of Ferromagnetic Induced Inhomogeneity in Excess Conductivity of $\mathrm{YBa}_{2} \mathrm{Cu}_{3} \mathrm{O}_{7-\delta+x} \mathrm{CoFe}_{2} \mathrm{O}_{4}$ Composite. J. Mater. Sci. Eng, 2012, Vol. 1. No. 115.

[27]Schilling, A., Cantoni, M., Guo, J. D. and Ott, H. R. SUPERCONDUCTIVITY ABOVE 130-K IN THE HG-BA-CA-CU-O SYSTEM. Nature, 1993, Vol. 363. No. 6424. 56-58. 
[28]Shams, G. A., Cochrane, J. W. and Russell, G. J. Thermal transport in polycrystalline YBa2Cu3O7-delta,Y2BaCuO5 and melt-processed YBa2Cu3O7-delta materials. Physica C, 2001, Vol. 351. No. 4. 449-465.

[29]Shih, W. Y., Ebner, C. and Stroud, D. FRUSTRATION AND DISORDER IN GRANULAR SUPERCONDUCTORS. Physical Review B, 1984, Vol. 30. No. 1. 134-144.

[30]Strickland, N. M., Long, N. J., Talantsev, E. F., Hoefakker, P., Xia, J., Rupich, M. W., Kodenkandath, T., Zhang, W., Li, X. and Huang, Y. Enhanced flux pinning by $\mathrm{BaZrO}_{3}$ nanoparticles in metal-organic deposited YBCO second-generation HTS wire. Physica C: Superconductivity, 2008, Vol. 468. No. 3. 183-189.

[31]Suan, M. S. M. and Johan, M. R. Synthesis of Al2O3 nanoparticles highly distributed in $\mathrm{YBa} 2 \mathrm{Cu} 3 \mathrm{O} 7$ superconductor by citrate-nitrate auto-combustion reaction. Physica C, 2013, Vol. 492. No. 49-54.

[32]Repeated Author. Microhardness of $\mathrm{Al}_{2} \mathrm{O}_{3}$ nanoparticles added $\mathrm{YBa}_{2} \mathrm{Cu}_{3} \mathrm{O}_{7-\delta}$ superconductor prepared using auto-combustion reaction. Materials Research Innovations, 2014, Vol. 18. No. S6. S6-73-S6-77.

[33] Taylor, K.N.R., Russell, G.J., Harris, L.B., Vaile, R.A., Cochrane, J., Matthews, D., Puzzer, T., Sun, H.B., Bailey, A., Bosi, S. and LaRobina, M. Y-Ba-Cu-O superconductors at the university of New South Wales. International Journal of Modern Physics B, 1987, Vol. 01. No. 02. 195-198.

[34] Varma, C. M. Erratum: Phenomenology of the normal state of $\mathrm{Cu}-\mathrm{O}$ high-temperature superconductors [Phys. Rev. Lett. 63, 1996 (1989)]. Physical Review Letters, 1990, Vol. 64. No. 4. 497-497.

[35]Velez, M., Martin, J. I., Villegas, J. E., Hoffmann, A., Gonzalez, E. M., Vicent, J. L. and Schuller, I. K. Superconducting vortex pinning with artificial magnetic nanostructures. Journal of Magnetism and Magnetic Materials, 2008, Vol. 320. No. 21. 2547-2562.

[36]Xu, C., Hu, A., Ichihara, M., Sakai, N., Izumi, M. and Hirabayashi, I. Enhanced flux pinning in $\mathrm{GdBaCuO}$ bulk superconductors by $\mathrm{Zr}$ dopants. Physica C: Superconductivity and its Applications, 2007, Vol. 463-465. No. 367-370. 
[37]Xu, Caixuan, Hu, Anming, Sakai, Naomichi, Izumi, Mitsuru and Hirabayashi, Izumi. Flux pinning properties and superconductivity of Gd-123 superconductor with addition of nanosized $\mathrm{SnO}_{2} / \mathrm{ZrO}_{2}$ particles. Physica C: Superconductivity and its Applications, 2006, Vol. 445-448. No. 357-360.

[38]Xu, Y., Hu, A., Xu, C., Sakai, N., Hirabayashi, I. and Izumi, M. Effect of $\mathrm{ZrO}_{2}$ and ZnO nanoparticles inclusions on superconductive properties of the melt-processed $\mathrm{GdBa}_{2} \mathrm{Cu}_{3} \mathrm{O}_{7-\delta}$ bulk superconductor. Physica C: Superconductivity, 2008, Vol. 468. No. 15-20. 1363-1365.

\section{How to cite this article:}

Shams G. A. and Mahmoodinezhad A. Influence of magnetic fields on the critical temperature of bulk YBCO superconductor with nanometer-sized $\mathrm{al}_{2} \mathrm{O}_{3}$ inclusions. J. Fundam. Appl. Sci., 2016, $8(2 S), 211-227$. 